\title{
Combination treatment with paclitaxel and doxorubicin inhibits growth of human esophageal squamous cancer cells by inactivation of Akt
}

\author{
HWAN HEE LEE ${ }^{1}$, SHUAI YE ${ }^{2}$, XIU JUAN LI ${ }^{1}$, KWANG BOK LEE ${ }^{2}$, MAN HEE PARK ${ }^{3}$ and SOO MI KIM $^{1}$ \\ Departments of ${ }^{1}$ Physiology and ${ }^{2}$ Orthopedic Surgery, Institute for Medical Sciences, Chonbuk National University \\ Medical School and Hospital, Jeonju, Jeonbuk; ${ }^{3}$ Catholic University of Pusan, Busan, Republic of Korea
}

Received October 9, 2013; Accepted November 4, 2013

DOI: $10.3892 /$ or.2013.2851

\begin{abstract}
Despite the fact that paclitaxel and doxorubicin are widely used as chemotherapy agents against several types of cancer, their combined effects on esophageal squamous cell carcinoma (ESCC) have never been fully elucidated. The present study was designed to investigate the biological effects of paclitaxel and doxorubicin in ESCC cells. Combination treatment with paclitaxel and doxorubicin significantly inhibited the proliferation of TE-12 cells in a dose-and timedependent manner compared to treatment with paclitaxel or doxorubicin alone. FACS analysis showed that the percentage of cells in the G2/M phase was significantly increased at $12 \mathrm{~h}$ after treatment with the combination. Increased $\mathrm{p}$-cdc2, p-Wee1 and p53 protein levels were observed, while Akt activation was suppressed by combination treatment with paclitaxel and doxorubicin. In addition, treatment with paclitaxel plus doxorubicin significantly increased apoptosis as indicated by increased cleaved poly(ADP-ribose) polymerase and cleaved caspase- 7 and -9 levels. These results suggest that combination treatment with paclitaxel and doxorubicin induced G2/M cell cycle arrest and apoptosis in human ESCC cells by suppressing Akt activity. These findings highlight the potent apoptotic effect of combination therapy with paclitaxel and doxorubicin in ESCC cells and the potential clinical benefits of these two drugs in esophageal cancer.
\end{abstract}

\section{Introduction}

Esophageal squamous cell carcinoma (ESCC) remains a major worldwide gastrointestinal tract malignancy. Although the incidence of ESCC has decreased in Western countries, it

Correspondence to: Dr Soo Mi Kim, Department of Physiology, Chonbuk National University Medical School and Hospital, 2-20 Keum-Am-Dong-San, Jeonju, Jeonbuk 561-180, Republic of Korea E-mail: soomikim@jbnu.ac.kr

Key words: esophageal cancer, paclitaxel, doxorubicin, apoptosis, Akt, cell cycle is still a major public health problem worldwide $(1,2)$. Since ESCC usually occurs in the middle and upper part of the esophagus, the risks of surgery often outweigh the benefits, making this a fatal cancer $(3,4)$. Due to its poor prognosis, major efforts have been undertaken to discover new therapies for ESCC (5).

Many ESCC patients receive chemotherapy regardless of their TNM-stage due to a high perioperative risk (3). Consequently, chemotherapy has been widely used in ESCC patients and has proven benefits. Paclitaxel, platinums and fluoropyrimidines are all used for the treatment of ESCC (1). Paclitaxel arrests the cell cycle by interfering with the microtubular network during cell division $(1,3)$. It is commonly administered in patients with breast, lung or prostate cancer and those with melanoma due to its cytotoxic effect $(1,6-9)$. Although platinums and fluoropyrimidines have historically been used to treat gastroesophageal cancer, paclitaxel alone or in combination with other agents such as $5 \mathrm{FU}$ or cisplatin is a current therapy (1). Studies have suggested that paclitaxel is efficacious in patients with ESCC and its use results in improvements in clinical outcomes $(1,10,11)$. However, patients who are treated with paclitaxel often relapse or do not respond to the treatment. In vitro experiments have shown that paclitaxel induces apoptosis in ESCC cells but different sensitivities were observed, suggesting that responses to paclitaxel may vary (12). Higher dosages of paclitaxel can induce toxicity in kidney and liver.

Doxorubicin is an effective cytotoxic anticancer agent and has been used for the treatment of variety of malignancies (13). Doxorubicin binds to DNA-associated enzymes and intercalates between DNA base pairs, ultimately resulting in DNA damage (13) that leads to apoptosis by inhibiting the cell cycle and nuclear DNA polymerase $(13,14)$. Although doxorubicin is widely used in the treatment of several types of cancer, its clinical use is limited by severe dose-dependent toxicities. At higher dosages, both paclitaxel and doxorubicin are toxic to many organs including the kidney, heart, brain and liver. Thus, great care must be taken in the use of these agents.

Combination chemotherapy has been reported to be more effective than single agent therapy. However, data on combination chemotherapy in ESCC cells are limited, especially for paclitaxel and doxorubicin. Here, we examined a modestly 
toxic combination of paclitaxel and doxorubicin to determine whether they have more significant biological effects together than as single agents alone in ESCC cells. We report that the combination treatment of paclitaxel and doxorubicin enhanced the induction of $\mathrm{G} 2 / \mathrm{M}$ cell cycle arrest and apoptosis in human ESCC cells by suppressing Akt activity. These findings emphasize the powerful apoptotic effect of combination therapy with paclitaxel and doxorubicin in ESCC cells and the potential clinical usefulness of these two drugs in esophageal cancer.

\section{Materials and methods}

Cell culture and reagents. Human esophageal cancer cell line TE-12 was obtained from Dr Izzo (University of Texas M.D. Anderson Cancer Center, Houston, TX, USA). These cancer cells were maintained as a monolayer in 100-mm dishes (BD Biosciences, Cockeysville, MD, USA) in DMEM-F12 medium (Gibco, Grand Island, NY, USA) supplemented with $10 \%$ fetal bovine serum (FBS) (Gibco), $100 \mathrm{mg} / \mathrm{ml}$ streptomycin and $100 \mathrm{IU} / \mathrm{ml}$ penicillin (Gibco) under standard conditions at $37^{\circ} \mathrm{C}$ in a $5 \% \mathrm{CO}_{2}$ humidified atmosphere. Paclitaxel and doxorubicin were obtained from Sigma-Chemical Co. (St. Louis, MO, USA) dissolved in dimethysulphoxide (DMSO; SigmaChemical). Further dilutions were performed in cell culture media and DMSO was used as a vehicle control. Cyclin B1, p-cdc2, p-Wee1, cleaved caspase-7, cleaved caspase-9, cleaved poly(ADP-ribose) polymerase (PARP), p-PTEN and p-Akt antibodies were obtained from Cell Signaling Technology (Danvers, MA, USA) and cdc2, Akt, p53 and $\beta$-actin antibodies were obtained from Santa Cruz Biotechnology Inc. (Dallas, TX, USA).

MTT assay. The cytotoxicity was monitored using the 3-(4,5-dimethylthiazol-2-yl)-2,5-diphenyltetrazolium bromide (MTT) assay as previously described (6). Briefly, cells (1x10 $)$ were seeded in 96-well plates (BD Biosciences, San Jose, CA, USA) containing $100 \mu \mathrm{l}$ of DMEM-F12 medium. After 24-h incubation under standard conditions, the cells were treated with various concentrations of paclitaxel and/or doxorubicin. After $72 \mathrm{~h}, 50 \mu \mathrm{l}$ of MTT (2 mg/ml PBS) was added and the plates were incubated for an additional $3 \mathrm{~h}$. The medium was aspirated off, and $200 \mu \mathrm{l}$ of DMSO was added. The optical density was assessed at a wavelength of $540 \mathrm{~nm}$ using a scanning multiwall spectrophotometer (SpectraMAX 340; Molecular Devices Co., Sunnyvale, CA, USA).

PI staining for cell cycle analysis. Cell sample preparation and PI staining were performed according to the manufacturer's protocol. Briefly, $1 \times 10^{6}$ cells were incubated with or without various concentrations of paclitaxel and/or doxorubicin. Cells were washed with PBS and the nuclei were stained with PI (Sigma Chemical) as previously described (6). The cell cycle distribution was measured with a FACStar flow cytometer (Becton-Dickinson, San Jose, CA, USA) and analyzed using Becton-Dickinson software (Lysis II and CellFit).

Immunoblot assay analysis. TE-12 human esophageal cancer cells were plated and allowed to attach for $24 \mathrm{~h}$. Paclitaxel and doxorubicin were added to cell cultures at various concentrations for 24-72 h. Cells with or without paclitaxel and doxorubicin were harvested and suspended in lysis buffer (Intron Biotechnology, Inc.). Extracts were incubated on ice for $10 \mathrm{~min}$ and centrifuged at $13,200 \mathrm{rpm}$ for $20 \mathrm{~min}$ at $4^{\circ} \mathrm{C}$. After centrifuging, the supernatant was collected. The protein concentration was determined using a BSA Protein Assay kit (Pierce, Rockford, IL, USA). Whole lysate was resolved on an SDS-PAGE gel and transferred to a PVDF membrane (Bio-Rad Laboratories, Hercules, CA, USA). Membranes were probed with specific primary antibodies and then with peroxidaseconjugated secondary antibodies. The bands were visualized with an Enhanced chemiluminescence kit (Amersham Health, Arlington Heights, IL, USA). Cyclin B1, p53, cdc2, p-cdc2, p-Wee1, cleaved caspase-7, cleaved caspase-9, cleaved PARP, p-Akt, Akt and $\beta$-actin antibodies were used.

Statistical analysis. Statistical analysis was performed using Student's t-test or one-way analysis of variance (ANOVA) where appropriate. All experiments were repeated more than three times and results are expressed as means \pm SE. P-values of $<0.05$ were considered to indicate statistically significant results.

\section{Results}

Growth inhibition of TE-12 cells by paclitaxel and doxorubicin. TE-12 esophageal squamous carcinoma cells were exposed to paclitaxel and doxorubicin in varying concentrations for $72 \mathrm{~h}$. As shown in Fig. 1, paclitaxel and doxorubicin inhibited cell viability in a dose-dependent manner. Since the $\mathrm{IC}_{50}$ of paclitaxel and doxorubicin was $20-25$ and 250-300 nM, respectively, we performed combination treatment experiments with $10 \mathrm{nM}$ paclitaxel and $125 \mathrm{nM}$ doxorubicin. Combination treatment with paclitaxel $(10 \mathrm{nM})$ and doxorubicin $(125 \mathrm{nM})$ resulted in significant growth inhibition (60-80\% at $72 \mathrm{~h}$ ) of TE-12 cells compared with either agent alone. These results suggest that combination treatment with paclitaxel and doxorubicin effectively inhibits the growth of esophageal squamous carcinoma cells.

Inhibition of colony formation by paclitaxel and doxorubicin. A soft agar cloning assay was performed to test the effect of paclitaxel and doxorubicin on TE-12 cell in vitro colony formation. Treatment with paclitaxel $(10 \mathrm{nM})$ and doxorubicin $(150 \mathrm{nM})$ resulted in significantly greater inhibition of colony formation in TE-12 cells when compared to treatment with a single agent (Fig. 2).

\section{Paclitaxel and doxorubicin affect cell cycle progression. FACS} was used to investigate the effect of paclitaxel and doxorubicin on cell cycle progression in esophageal squamous carcinoma cells. The cell cycle distribution was measured at $12 \mathrm{~h}$ after treatment with paclitaxel $(10 \mathrm{nM})$ alone, doxorubicin $(150 \mathrm{nM})$ alone, and paclitaxel $(10 \mathrm{nM})$ plus doxorubicin $(150 \mathrm{nM})$. As shown in Fig. 3A, significant accumulation of cells in G2/M phase was observed after exposure to paclitaxel and doxorubicin. Combination treatment with paclitaxel and doxorubicin significantly increased the percentage of TE-12 cells in the $\mathrm{G} 2 / \mathrm{M}$ (G1 percentage 54.22, $\mathrm{P}<0.05)$ compared to control (G1 percentage 26.21). We further investigated alterations in cell 


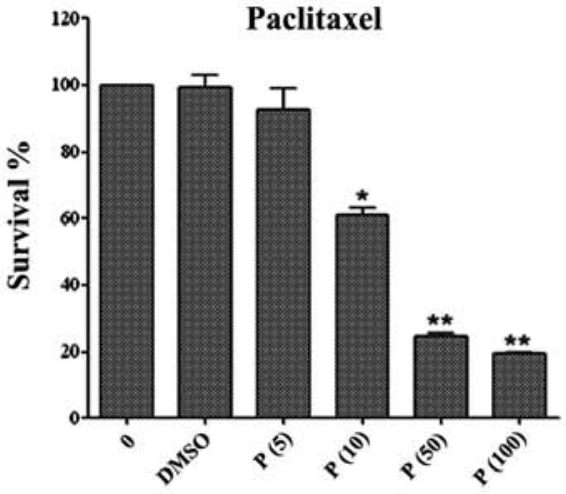

Concentration of drug (nM)

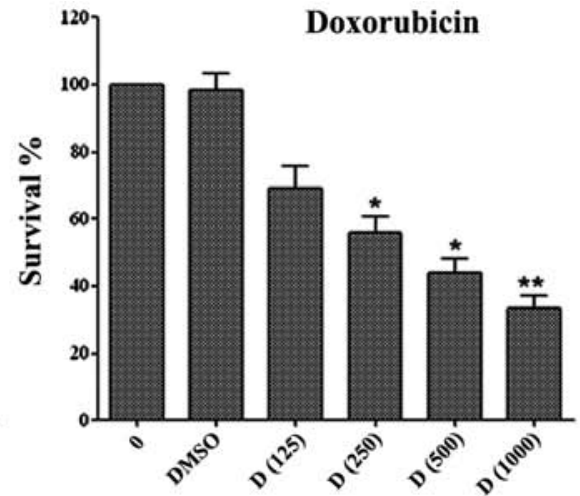

Concentration of drug $(\mathrm{nM})$

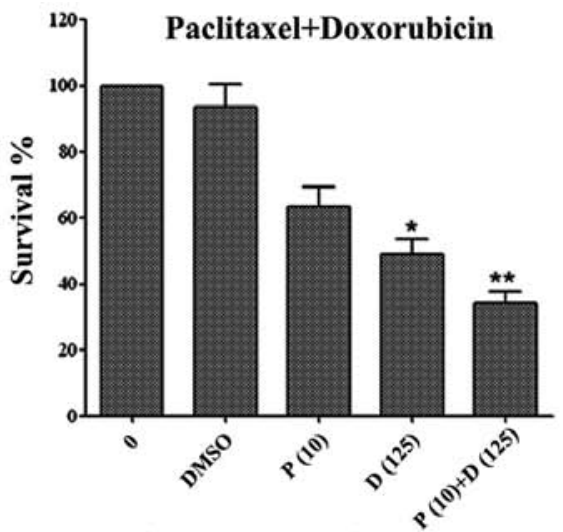

Concentration of drug (nM)

Figure 1. MTT assay measuring the effects of paclitaxel and doxorubicin alone or in combination on growth of TE-12 cells. Logarithmically growing TE-12 cells were treated with various concentrations of paclitaxel and doxorubicin for $72 \mathrm{~h}$. P, paclitaxel; D, doxorubicin. Data are expressed as percentage of control. Each point represents the means $( \pm \mathrm{SE})$ of at least three independent experiments. ${ }^{*} \mathrm{P}<0.05$ and ${ }^{* *} \mathrm{P}<0.001$ when compared to the control.

A
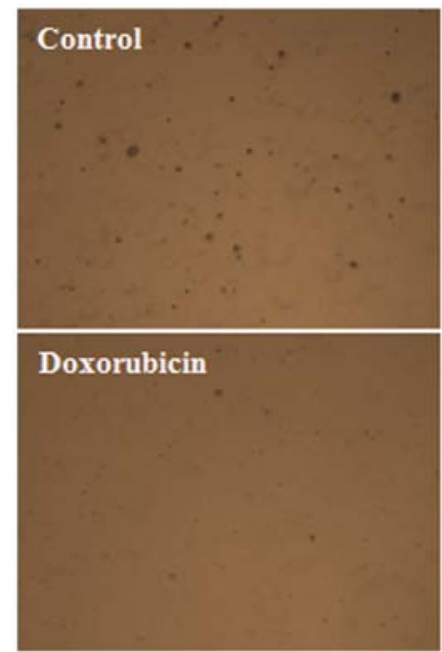

B

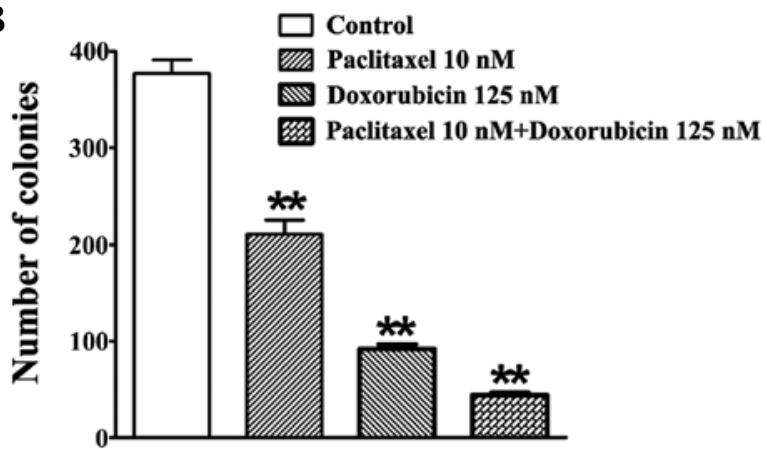

Figure 2. Colony formation assay. Paclitaxel and doxorubicin, either alone or in combination, inhibited colony formation in TE-12 cells. The combination of paclitaxel and doxorubicin significantly inhibited the colony formation ability of ESCC cells. The assays were terminated at 2 weeks and the colony numbers were counted under the microscope. ${ }^{* *} \mathrm{P}<0.001$ when compared to the control.

cycle regulatory proteins following paclitaxel and doxorubicin treatment. p-cdc2 and p-Weel protein levels were significantly increased by paclitaxel $(10 \mathrm{nM})$ and combination treatment
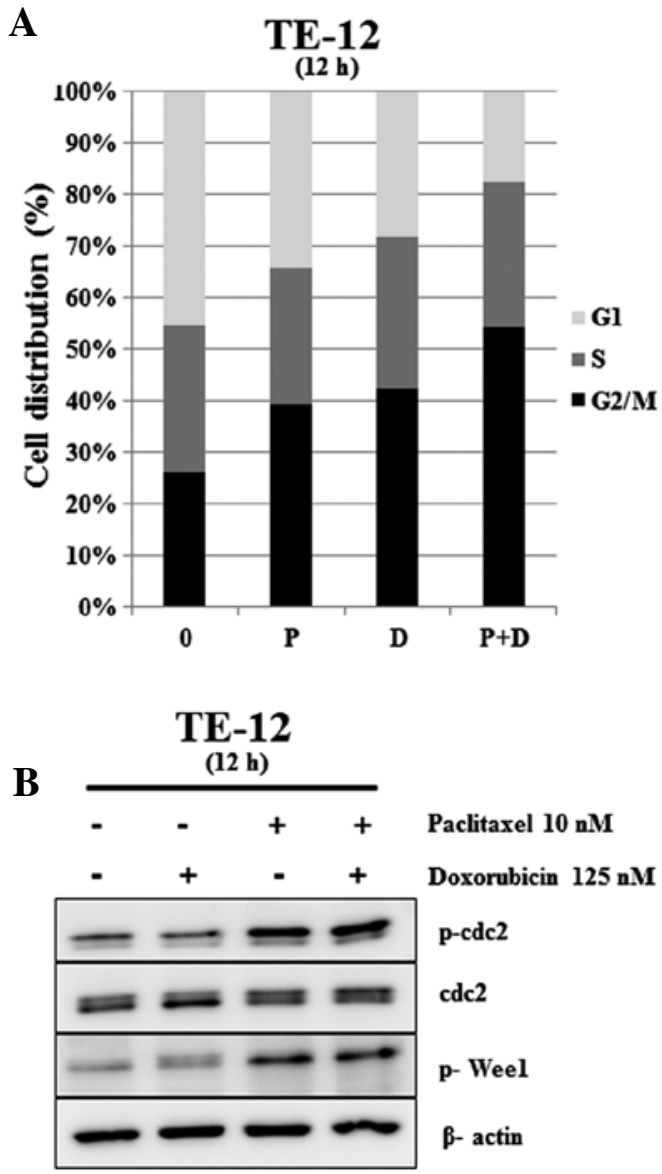

Figure 3. Cell cycle analysis of TE-12 cells treated with paclitaxel and doxorubicin, either alone or in combination. TE-12 cells were incubated for $12 \mathrm{~h}$ with $10 \mathrm{nM}$ paclitaxel and $125 \mathrm{nM}$ doxorubicin. (A) Cell cycle distribution was calculated as the percentage of cells in the G1, S and G2/M phase. P, paclitaxel; D, doxorubicin. (B) G2/M phase related proteins (p-cdc2, cdc2 and $p$-Wee1) in TE-12 cells treated with paclitaxel and doxorubicin, either alone or in combination, were measured by western blot analysis. $\beta$-actin was used as an internal control.

with paclitaxel $(10 \mathrm{nM})$ and doxorubicin $(125 \mathrm{nM})$, while no change was observed with doxorubicin treatment alone. Levels of the cyclin-dependent kinase inhibitor protein p53 increased 


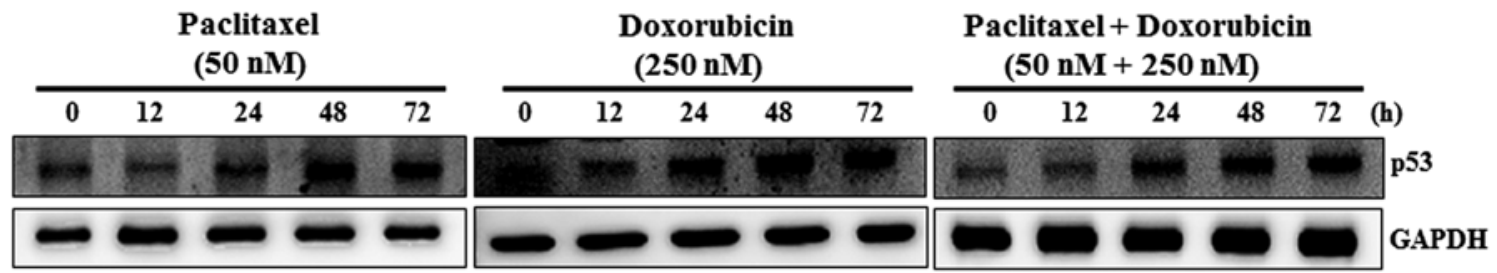

Figure 4. Effects of paclitaxel and doxorubicin on a cyclin-dependent kinase inhibitor in ESCC cells. p53 was measured by western blot analysis in TE-12 cells after treatment with paclitaxel and doxorubicin, either alone or in combination. Cells were harvested at $0,12,24,48$ and $72 \mathrm{~h}$ and immunoblotted with p53 antibodies. GAPDH was used as an internal control.

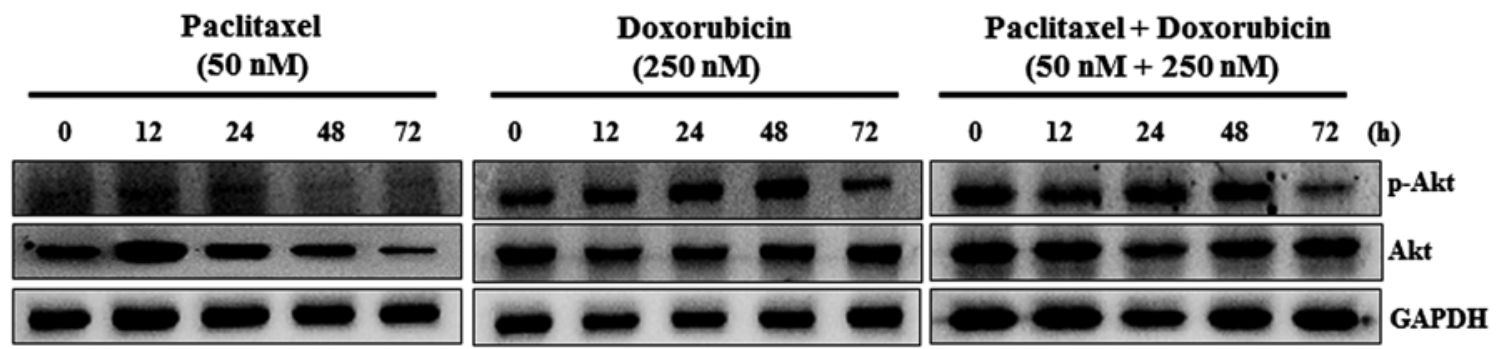

Figure 5. Effects of paclitaxel and doxorubicin on the Akt signaling pathway in ESCC cells. P-Akt and Akt were measured by western blots in TE-12 cells after treatment with paclitaxel and doxorubicin, either alone or in combination. Cells were harvested at $0,12,24,48$ and $72 \mathrm{~h}$ and immunoblotted with the indicated antibodies. GAPDH was used as an internal control.

with the concentration of paclitaxel $(50 \mathrm{nM})$ and doxorubicin $(250 \mathrm{nM})$ in a time-dependent manner (Fig. 4). Combination treatment with paclitaxel $(50 \mathrm{nM})$ and doxorubicin $(250 \mathrm{nM})$ also induced p53 protein expression in a time-dependent manner. These results indicate that combination treatment with paclitaxel and doxorubicin leads to a significant increase in the $\mathrm{G} 2 / \mathrm{M}$ population in esophageal squamous carcinoma cells.

Paclitaxel and doxorubicin suppress the phosphorylation of Akt. Cells were treated with paclitaxel and doxorubicin and Akt protein expression was followed by western blot analysis. As shown in Fig. 5, paclitaxel (50 nM) significantly inhibited p-Akt and Akt protein levels in a time-dependent manner. Doxorubicin $(250 \mathrm{nM})$ significantly inhibited the expression of p-Akt at $72 \mathrm{~h}$, but no change was seen at other time-points. Doxorubicin did not affect Akt expression. Combination treatment with paclitaxel and doxorubicin significantly suppressed the expression of p-Akt at $72 \mathrm{~h}$, but no difference was observed at other time-points. Akt protein levels were not altered by combination treatment with paclitaxel and doxorubicin.

Treatment with paclitaxel and doxorubicin induces apoptosis. We measured cleaved caspase-9, cleaved PARP and cleaved caspase-7 protein levels in TE-12 cells. As shown in Fig. 6, treatment with paclitaxel or doxorubicin alone increased the expression of cleaved PARP, cleaved caspase-7 and cleaved caspase- 9 proteins. Treatment with the combination of paclitaxel $(10 \mathrm{nM})$ and doxorubicin $(125 \mathrm{nM})$ significantly increased the expression of cleaved PARP, cleaved caspase-7 and cleaved caspase-9. These data indicate that the combination of paclitaxel and doxorubicin induces more marked apoptotic cell death in TE-12 esophageal carcinoma cells than a single agent alone.

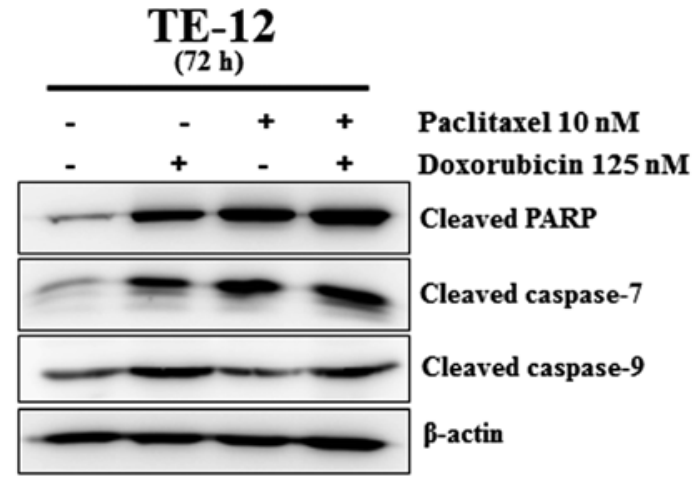

Figure 6. Effects of paclitaxel and doxorubicin on apoptosis-associated proteins in ESCC cells. Cleaved PARP, cleaved caspase-7 and cleaved caspase- 9 were measured by western blot analysis in TE- 12 cells after treatment with paclitaxel and doxorubicin, either alone or in combination. $\beta$-actin was used as an internal control.

\section{Discussion}

Despite significant reductions in esophageal cancer rates associated with lifestyle changes, ESCC remains the seventh leading cause of mortality in the USA and the sixth leading cause of mortality worldwide (15). It is one of the most fatal types of cancer and chronic irritation and inflammation appear to be the main cause of ESCC $(4,15)$. Since the morbidity and mortality associated with surgery for ESCC outweigh the benefits $(3,15)$, chemotherapy is largely employed to reduce tumor size, lower recurrence rates and prolong survival $(3,15)$. Although paclitaxel and doxorubicin are widely used as chemotherapy agents against several types of cancer, combination therapy with these agents has not been evaluated for ESCC. Here, we demonstrated, for the first time, the biological effects of combination therapy with paclitaxel and doxorubicin on ESCC cells. 
In the present study, we found that paclitaxel and doxorubicin significantly suppressed the proliferation of TE-12 cells in a dose-dependent manner. Concentrations of $10 \mathrm{nM}$ paclitaxel and $125 \mathrm{nM}$ doxorubicin were chosen for their efficacy in ESCC cells. Treatment with these two drugs in combination suppressed proliferation of TE-12 cells significantly more effectively than treatment with a single agent alone, suggesting that the two agents work substantially to suppress proliferation in ESCC cells. As expected from the cell viability assay, paclitaxel and doxorubicin inhibited colony formation in TE-12 cells. Combination treatment with paclitaxel and doxorubicin inhibited colony formation in TE-12 significantly more than treatment with a single agent alone. The antiproliferative effects of the drug combination were more marked in the colony formation assay than in the MTT assay, although the same dosages and cells were used. However, the inhibition patterns caused by treatment with paclitaxel and doxorubicin appeared similar. Therefore, combination therapy markedly increased the antiproliferative effects of these drugs in ESCC cells.

Several studies, including one of ours pertaining to esophageal adenocarcinoma, reported that paclitaxel inhibits cell replication by blocking progression beyond the late $\mathrm{G} 2$ and/or $\mathrm{M}$ phases of the cell cycle in various types of cancer $(1,12,16-18)$. Zimmermann et al (19) also demonstrated that doxorubicin induced G2/M cell cycle arrest secondary to DNA damage. In agreement with previous reports, we observed $\mathrm{G} 2 / \mathrm{M}$ cell cycle arrest induced by paclitaxel and doxorubicin in TE- 12 cells. The proportion of the cell population in $\mathrm{G} 2 / \mathrm{M}$ was increased by paclitaxel and doxorubicin treatment. Combination treatment with these two drugs significantly increased the proportion of the cell population in $\mathrm{G} 2 / \mathrm{M}$. In addition, G2/M phase-related proteins $\mathrm{p}$-cdc2 and p-Wee1 were also significantly induced by combination treatment with these two drugs. Expression of the cyclin-dependent kinase inhibitor p53 was increased by treatment with paclitaxel and doxorubicin in a time-dependent manner. Similar to treatment with either agent alone, combination treatment with these two drugs significantly increased expression of p53 in a time-dependent manner. Collectively, our findings strongly suggest that combination therapy with paclitaxel and doxorubicin significantly induces marked G2/M cell cycle arrest in ESCC cells.

The PI3K/Akt pathway plays an important role in controlling cell proliferation and increased PI3K/Akt activity has been observed in several types of cancer including ESCC (20-23). A recent study also linked mutation in the PI3K/ Akt pathway to prognosis of patients with ESCC (24). A few studies have concluded that paclitaxel induces apoptosis through the suppression of the Akt pathway in $\operatorname{ESCC}(25,26)$. Doxorubicin induced apoptosis in breast cancer through the suppression of Akt signaling (27). Yu et al (28) reported that doxorubicin induced apoptosis in lung cancer via modulation of the PI3K/Akt signaling pathway. However, Akt modulation mediated by doxorubicin has not been investigated in ESCC cells. Therefore, we explored changes in the levels of Akt and p-Akt induced by treatment with doxorubicin alone and in combination with paclitaxel in ESCC cells. In the present study, p-Akt protein levels were significantly diminished in a time-dependent manner by single-agent treatment with paclitaxel or doxorubicin and combination treatment further decreased p-Akt levels. Consistent with its behavior in other types of cancer, doxorubicin also suppressed the expression of Akt in ESCC cells, although this effect only became significant after $72 \mathrm{~h}$. Levels of the apoptosis-associated proteins cleaved PARP, cleaved caspase-7 and cleaved caspase- 9 were increased by single-agent treatment with paclitaxel and doxorubicin. Combination treatment with paclitaxel and doxorubicin strongly induced the expression of these apoptosis-associated proteins. These results indicate that apoptosis induced by combination treatment with paclitaxel and doxorubicin may be affected by the caspase-dependent pathway.

Our experiments suggest that paclitaxel and doxorubicin synergistically inhibit proliferation of ESCC cells by inducing G2/M cell cycle arrest and apoptosis. Apoptosis induced by treatment with paclitaxel and doxorubicin may proceed secondary to the suppression of Akt signaling, although further experiments are required to investigate the signaling mechanisms involved. Based on our findings, we believe that combination therapy with paclitaxel and doxorubicin may prove to be a successful approach for the treatment of ESCC.

\section{Acknowledgements}

The present study was supported by research funds from the Chonbuk National University in 2012 and by the Basic Science Research Program through the National Research Foundation of Korea (NRF) funded by the Ministry of Education, Science and Technology (20110014864 and 2012R1A1A2005729).

\section{References}

1. Jimenez P, Pathak A and Phan AT: The role of taxanes in the management of gastroesophageal cancer. J Gastrointest Oncol 2: 240-249, 2011.

2. Ajani JA, Barthel JS, Bekaii-Saab T, et al: Esophageal cancer. J Natl Compr Canc Netw 6: 818-849, 2008.

3. Wolf MC, Stahl M, Krause BJ, et al: Curative treatment of oesophageal carcinoma: current options and future developments. Radiat Oncol 6: 55, 2011.

4. Jemal A, Center MM, DeSantis C and Ward EM: Global patterns of cancer incidence and mortality rates and trends. Cancer Epidemiol Biomarkers Prev 19: 1893-1907, 2010.

5. Wright JC: Update in cancer chemotherapy: gastrointestinal cancer, cancer of the small intestines, gallbladder, liver, and esophagus. J Natl Med Assoc 78: 753-766, 1986.

6. Kim SJ, Chung MJ, Kim JS, et al: Deciphering the role of paclitaxel in the SKGT4 human esophageal adenocarcinoma cell line. Int J Oncol 39: 1587-1591, 2011.

7. Legha SS, Ring S, Papadopoulos N, Raber M and Benjamin RS: A phase II trial of taxol in metastatic melanoma. Cancer 65: 2478-2481, 1990.

8. Holmes FA, Walters RS, Theriault RL, et al: Phase II trial of taxol, an active drug in the treatment of metastatic breast cancer. J Natl Cancer Inst 83: 1797-1805, 1991.

9. Einzig AI, Wiernik PH, Sasloff J, Runowicz CD and Goldberg GL: Phase II study and long-term follow-up of patients treated with taxol for advanced ovarian adenocarcinoma. J Clin Oncol 10: $1748-1753,1992$.

10. van Steenbergen LN, Elferink MA, Krijnen P, et al: Improved survival of colon cancer due to improved treatment and detection: a nationwide population-based study in The Netherlands 1989-2006. Annal Oncol 21: 2206-2212, 2010.

11. Ajani J: Therapy of localized esophageal cancer: it is time to reengineer our investigative strategies. Onkologie 31: 360-361, 2008.

12. Faried A, Faried LS, Kimura H, et al: Differential sensitivity of paclitaxel-induced apoptosis in human esophageal squamous cell carcinoma cell lines. Cancer Chemother Pharmacol 57: 301-308, 2006 . 
13. Tacar O, Sriamornsak P and Dass CR: Doxorubicin: an update on anticancer molecular action, toxicity and novel drug delivery systems. J Pharm Pharmacol 65: 157-170, 2013.

14. Buchholz TA, Stivers DN, Stec J, et al: Global gene expression changes during neoadjuvant chemotherapy for human breast cancer. Cancer J 8: 461-468, 2002.

15. Enzinger PC and Mayer RJ: Esophageal cancer. New Engl J Med 349: 2241-2252, 2003.

16. Rowinsky EK and Donehower RC: The clinical pharmacology of paclitaxel (Taxol). Semin Oncol 20: 16-25, 1993

17. Park KW, Kang SH, Park KH, et al: Sirolimus- vs. paclitaxeleluting stents for the treatment of unprotected left main coronary artery stenosis: complete 2-year follow-up of a two-center registry. Int J Cardiol 151: 89-95, 2011.

18. Toiyama Y, Tanaka K, Konishi N, et al: Administration sequencedependent antitumor effects of paclitaxel and 5-fluorouracil in the human gastric cancer cell line MKN45. Cancer Chemother Pharmacol 57: 368-375, 2006.

19. Zimmermann M, Arachchige-Don AS, Donaldson MS, Dallapiazza RF, Cowan CE and Horne MC: Elevated cyclin G2 expression intersects with DNA damage checkpoint signaling and is required for a potent $\mathrm{G} 2 / \mathrm{M}$ checkpoint arrest response to doxorubicin. J Biol Chem 287: 22838-22853, 2012.

20. Kim AH, Khursigara G, Sun X, Franke TF and Chao MV: Akt phosphorylates and negatively regulates apoptosis signalregulating kinase 1. Mol Cell Biol 21: 893-901, 2001.

21. Zhang HB, Lu P, Guo QY, Zhang ZH and Meng XY: Baicalein induces apoptosis in esophageal squamous cell carcinoma cells through modulation of the PI3K/Akt pathway. Oncol Lett 5: 722-728, 2013.

22. Lin ML, Lu YC, Chen HY, Lee CC, Chung JG and Chen SS: Suppressing the formation of lipid raft-associated Rac1/PI3K/Akt signaling complexes by curcumin inhibits SDF-1alpha-induced invasion of human esophageal carcinoma cells. Mol Carcinog Nov 28, 2012 (Epub ahead of print). doi: 10.1002/mc.21984.
23. Gen Y, Yasui K, Nishikawa T and Yoshikawa T: SOX2 promotes tumor growth of esophageal squamous cell carcinoma through the AKT/mammalian target of rapamycin complex 1 signaling pathway. Cancer Sci 104: 810-816, 2013

24. Shigaki H, Baba Y, Watanabe M, et al: PIK3CA mutation is associated with a favorable prognosis among patients with curatively resected esophageal squamous cell carcinoma. Clin Cancer Res 19: 2451-2459, 2013.

25. Ou Y, Ma L, Ma L, et al: Overexpression of cyclin B1 antagonizes chemotherapeutic-induced apoptosis through PTEN/Akt pathway in human esophageal squamous cell carcinoma cells. Cancer Biol Ther 14: 45-55, 2013.

26. Nguyen DM, Chen GA, Reddy R, et al: Potentiation of paclitaxel cytotoxicity in lung and esophageal cancer cells by pharmacologic inhibition of the phosphoinositide 3-kinase/protein kinase B (Akt)-mediated signaling pathway. J Thorac Cardiovasc Surg 127: 365-375, 2004.

27. Chen Y, Sun Y, Chen L, et al: miRNA-200c increases the sensitivity of breast cancer cells to doxorubicin through the suppression of E-cadherin-mediated PTEN/Akt signaling. Mol Med Rep 7: 1579-1584, 2013.

28. Yu YH, Kuo HP, Hsieh HH, et al: Ganoderma tsugae induces $\mathrm{S}$ phase arrest and apoptosis in doxorubicin-resistant lung adenocarcinoma $\mathrm{H} 23 / 0.3$ cells via modulation of the PI3K/Akt signaling pathway. Evid Based Complement Alternat Med 2012: 371286, 2012. 